\title{
An Improved Nonhomogeneous Discrete Grey Model and Its Application
}

\author{
Jianming Jiang $\mathbb{D},{ }^{1}$ Yu Zhang, ${ }^{2}$ Changqing Liu $\mathbb{D},{ }^{1}$ and Wanli Xie ${ }^{3}$ \\ ${ }^{1}$ School of Mathematics and Statistics, Baise University, Baise 533000, China \\ ${ }^{2}$ Department of Mathematics, Nanjing University of Aeronautics and Astronautics, Nanjing 210016, China \\ ${ }^{3}$ Institute of EduInfo Science and Engineering, Nanjing Normal University, Nanjing 210097, China \\ Correspondence should be addressed to Changqing Liu; lcqing619@126.com
}

Received 20 February 2020; Accepted 16 April 2020; Published 8 May 2020

Academic Editor: Luis M. López-Ochoa

Copyright ( $\odot 2020$ Jianming Jiang et al. This is an open access article distributed under the Creative Commons Attribution License, which permits unrestricted use, distribution, and reproduction in any medium, provided the original work is properly cited.

In recent years, the nonhomogeneous grey model has received much attention owing to its flexibility and applicability of forecasting small samples. To improve further the prediction accuracy of the nonhomogeneous grey model, this paper is to introduce a new whitening equation with variable coefficient into the original nonhomogeneous grey model, which is abbreviated as $\operatorname{ONGM}(1,1, k, c)$. First of all, the detailed computational steps of the time response function of the novel model and the restored values of the raw data sequence are deduced through grey modelling techniques. Secondly, two empirical examples from the previous literature are conducted to prove the validity of the novel model. Finally, the novel model is applied to forecast natural gas demand of China, and the results show that the novel model has a better prediction performance compared with other commonly used grey models, including $\operatorname{GM}(1,1), \operatorname{DGM}(1,1), \operatorname{NGM}(1,1, k, c)$, and $\operatorname{NGBM}(1,1)$.

\section{Introduction}

Grey prediction model is one of the key branches in grey system theory [1], which has been widely used in various fields, including industry $[2,3]$, engineering $[4,5]$, and especially energy [6-8] on account of its abilities of sufficiently dealing with small sample-sized problems. Besides, many extensive models of the benchmark grey model have been developed in practical applications. For example, Xie and Liu [9] investigated the discrete grey model which is abbreviated as $\operatorname{DGM}(1,1)$; in their work, they also revealed the connection of $\operatorname{DGM}(1,1)$ to the traditional grey model. Kumar and Jain [10] utilized grey Markov, grey model with the rolling mechanism and singular spectrum analysis to forecast energy consumption in India. Hamzacebi et al. [11] presented an improved grey model through combining iterative and direct grey forecasting approaches in order to predict electricity demand of Turkey. Ding et al. [12] combined a new initial condition and rolling mechanism to improve the prediction performance of the traditional grey model and then employed the model to forecast electricity consumption of China. Chen et al. [13] put forward a grey approach to achieve time-series interval forecasting. Inspired by existing approaches to forecasting time-series intervals [13], Wu and Zhang [14] further optimized the grey method for forecasting time-series intervals. Ma and Liu [15] raised a novel time-delayed polynomial grey model and applied it to forecast natural gas consumption of China. Intharathirat et al. [16] used multivariate grey models to forecast municipal solid waste quantity in developing countries.

However, it should be noticed that these above models are all linear, which are unapplicable for nonlinear problems. As a result, Chen et al. [17] firstly propounded a novel nonlinear grey Bernoulli model by introducing the Bernoulli equation into the grey action quality. Afterward, Wang et al. [18] proposed an improved nonlinear grey Bernoulli model which is based on optimization of background value. Under the inspiration of the literature [18], Wu et al. [19] investigated a new approach to improve the prediction performance by simultaneously considering the new initial condition and dynamic background value. Nguyen et al. [20] 
proposed a nonlinear grey Bernoulli model based on Fourier transformation. Ma et al. [21] proposed a novel nonlinear multivariable grey Bernoulli model to forecast tourism income of China, among them. It is worth noting the accumulation appeared in these models is all integer-order accumulation that impairs the prediction accuracy of forecasting models. For this, Wu et al. [22] originally proposed a grey model with fractional-order accumulation. Later, they also proposed a novel fractional multivariate grey model to forecast electricity consumption of Shandong Province [23]. Considering that computation of the existing fractional accumulation is complicated, therefore, Ma et al. [24] introduced a novel definition of conformable fractional accumulation, which is helpful in developing grey forecasting models.

Besides, the nonhomogeneous grey model, proposed by Cui et al. [25], also has received extensive attention owing to its ease of construction and flexibility. Chen and $\mathrm{Yu}$ [26] proposed a novel nonhomogeneous grey model in which the grey action quality was replaced by $b t+c$. Meanwhile, $\mathrm{Ma}$ et al. [27] investigated a novel kernel regularized nonhomogeneous grey model. Zeng et al. [28] developed a selfadaptive intelligence grey model. Based on the aforementioned knowledge, this paper introduces a new whitening equation with variable coefficient into the nonhomogeneous grey model to further enhance the prediction ability of the traditional nonhomogeneous grey model, which is abbreviated $\operatorname{ONGM}(1,1, k, c)$. The novelties of this paper are drawn as follows: (i) a new whitening equation with variable coefficient is considered into the nonhomogeneous grey model; (2) the time response function and the restored values of the original series are deduced in detail; (3) the two empirical examples are used to validate the effectiveness of the novel model; and (4) the novel model is applied in natural gas demand of China.

The rest of this paper is organized as follows: Section 2 briefly depicts the $\operatorname{NGM}(1,1, k, c)$ model. In Section 3, the modelling procedure of the novel model is discussed in detail. Section 4 provides two empirical examples to validate the novel model. Section 5 utilizes the novel model to forecast natural gas demand of China, and the main conclusions are listed in Section 6.

\section{Description of the Traditional Nonhomogeneous Grey Model}

To further improve the prediction performance of the $\operatorname{NGM}(1,1)$ model, Chen and Yu presented the nonhomogeneous discrete grey model, namely, $\operatorname{NGM}(1,1, k, c)$. The computational steps of $\operatorname{NGM}(1,1, k, c)$ are described as follows.

Step 1. Assume a nonnegative series to be

$$
X^{(0)}=\left(x^{(0)}(1), x^{(0)}(2), \ldots, x^{(0)}(n)\right),
$$

and then the first-order accumulative generating operation $((1-\mathrm{AGO}))$ series is

$$
X^{(1)}=\left(x^{(1)}(1), x^{(1)}(2), \ldots, x^{(1)}(n)\right),
$$

where $x^{(0)}(k)=\sum_{i=1}^{k} x^{(0)}(1)$.

Step 2. The whitening equation of $\operatorname{NGM}(1,1, k, c)$ can be given by

$$
\frac{\mathrm{d} x^{(1)}(t)}{\mathrm{d} t}+a x^{(1)}(t)=b t+c
$$

where $a$ and $b t+c$ represent the development parameter and grey action quality, respectively.

Step 3. The discrete form of equation (3) is easily written as

$$
x^{(0)}(k)+a z^{(1)}(k)=b k+c
$$

where $z^{(1)}(k)$ is called the background value and $z^{(1)}(k)=0.5\left(x^{(1)}(k)+x^{(1)}(k-1)\right)$.

Step 4. The model parameters $a, b$, and $c$ can be estimated through the least square method; there is

$$
(a, b, c)^{T}=\left(B^{T} B\right)^{-1} B^{T} Y,
$$

where

$$
B=\left(\begin{array}{ccc}
-z^{(1)}(2) & 2 & 1 \\
-z^{(1)}(3) & 3 & 1 \\
\vdots & \vdots & \vdots \\
-z^{(1)}(n) & n & 1
\end{array}\right), Y=\left(x^{(0)}(2), x^{(0)}(3), \ldots, x^{(0)}(n)\right)^{T}
$$

Step 5. The time response function of equation (3) with $x^{(1)}(1)=x^{(0)}(1)$ is given by

$$
\hat{x}^{(1)}(k)=\left(x^{(0)}(1)-\frac{b}{a}-\frac{c}{a}+\frac{b}{a^{2}}\right) e^{-a(k-1)}+\frac{b}{a} t+\frac{c}{a}-\frac{b}{a^{2}} .
$$

Step 6. The restored value of $x^{(0)}(k), k=2,3, \cdots$, can be acquired by using the first-order inverse accumulative generating operation ( 1 - IAGO); there is

$$
\widehat{x}^{(0)}(k)=\widehat{x}^{(1)}(k)-\widehat{x}^{(1)}(k-1) \text {. }
$$

\section{Presentation of the ONGM $(1,1, k, c)$ Model}

Definition 1. Let $u>0$; the whitening equation of a novel grey forecasting model is

$$
\frac{\mathrm{d} x^{(1)}(t)}{\mathrm{d} t^{u}}+a x^{(1)}(t)=b t+c
$$

It is worth noting that equation (9) will be degenerated to equation (3) when $u=1$. In other words, the novel model is a more general form of $\operatorname{NGM}(1,1, k, c)$. 
Theorem 1. The discrete formula of the whitening equation of the novel model is

$$
\begin{aligned}
x^{(0)}(k)+a z_{1}^{(1)}(k)= & b\left(\frac{u}{u+1}\left(k^{u+1}-(k-1)^{u+1}\right)\right) \\
& +c\left(k^{u}-(k-1)^{u}\right),
\end{aligned}
$$

where $z_{1}^{(1)}(k)=1 / 2\left(u k^{u-1}+u(k-1)^{u-1} x^{(0)}(k-1)\right)$.

Proof. According to equation (9), it is easily yielded

$$
\frac{\mathrm{d} x^{(1)}(t)}{\mathrm{d} t}+a u t^{u-1} x^{(1)}(t)=b u t^{u}+c u t^{u-1} .
$$

Integrating both sides of equation (11) over the interval $[k-1, k]$,

$$
\begin{aligned}
& \int_{k-1}^{k} \mathrm{~d} x^{(1)}(t)+\int_{k-1}^{k} a u t^{u-1} x^{(1)}(t) \mathrm{d} t=\int_{k-1}^{k} b u t^{u} \mathrm{~d} t \\
& +\int_{k-1}^{k} c u t^{u-1} \mathrm{~d} t .
\end{aligned}
$$

Using the two-point trapezoidal formula, equation (12) becomes

$$
\begin{aligned}
x^{(0)}(k)+a z_{1}^{(1)}(k)= & b\left(\frac{u}{u+1}\left(k^{u+1}-(k-1)^{u+1}\right)\right) \\
& +c\left(k^{u}-(k-1)^{u}\right) .
\end{aligned}
$$

This completes the proof.

Similar to Section 2, the model parameters of the novel model can be given by

$$
(a, b, c)^{T}=\left(H^{T} H\right)^{-1} H^{T} Y
$$

where

$$
H=\left(\begin{array}{ccc}
-z_{1}^{(1)}(2) & \frac{u}{u+1}\left(2^{u+1}-1^{u+1}\right) & 2^{u}-1^{u} \\
-z_{1}^{(1)}(3) & \frac{u}{u+1}\left(3^{u+1}-2^{u+1}\right) & 3^{u}-2^{u} \\
\vdots & \vdots & \vdots \\
-z_{1}^{(1)}(n) \frac{u}{u+1}\left(n^{u+1}-(n-1)^{u+1}\right) & n^{u}-(n-1)^{u}
\end{array}\right) .
$$

Theorem 2. Given the model parameters $a, b$, and $c$ of the novel model, the corresponding time response function can be given by

$$
x^{(1)}(t)=e^{-a t^{u}}\left(e^{a} x^{(0)}(1)+b \int_{1}^{k} f(u, \tau) \mathrm{d} \tau+\frac{c}{a}\left(e^{a t^{u}}-e^{a}\right)\right) .
$$

Proof. Multiply both sides of equation (9) by $e^{a t^{u}}$ :

$$
e^{a t^{u}}\left(\frac{\mathrm{d} x^{(1)}(t)}{\mathrm{d} t}+a u t^{u-1} x^{(1)}(t)\right)=e^{a t^{u}}\left(b u t^{u}+c u t^{u-1}\right) .
$$

That is,

$$
\frac{\mathrm{d} e^{a t^{u}} x^{(1)}(t)}{\mathrm{d} t}=e^{a t^{u}}\left(b u t^{u}+c u t^{u-1}\right)
$$
$[1, t]$,

Integrating both sides of equation (18) over the interval

$$
\int_{1}^{t} \mathrm{~d}\left(e^{a \tau^{u}} x^{(1)}(\tau)\right)=\int_{1}^{t} e^{a \tau^{u}}\left(b u \tau^{u}+c u \tau^{u-1}\right) \mathrm{d} \tau .
$$

Furthermore, by simplifying equation (19),

$$
x^{(1)}(t)=e^{-a t^{u}}\left(e^{a} x^{(0)}(1)+b \int_{1}^{k} f(u, \tau) \mathrm{d} \tau+\frac{c}{a}\left(e^{a t^{u}}-e^{a}\right)\right) \text {, }
$$

where $f(u, t)=e^{a t^{u}}$.

In particular, $f(u, t)$ can be approximately calculated by the following equation:

$$
\int_{1}^{t} f(u, \tau) \mathrm{d} \tau \approx \sum_{i=1}^{1000} \triangle_{i} f\left(u, \varepsilon_{i}\right)
$$

where $\triangle_{i}$ is taken as 0.001 for any $i$.

It is proved.

\section{Validation of the ONGM $(1,1, k, c)$ Model}

This section provides two empirical examples from the previous literature to demonstrate the effectiveness of the novel model; the competitive models including the traditional grey model $(\operatorname{GM}(1,1))$, the discrete grey model (DGM $(1,1))$, the nonlinear grey Bernoulli model $\operatorname{NGBM}(1,1))$, and the $\operatorname{NGM}(1,1, k, c)$ model are established thereof. To assess the prediction accuracies of these models, two statistical indices are used, which are the root mean square error (RMSE) and the mean absolute percentage error (MAPE):

$$
\begin{gathered}
\text { RMSE }=\sqrt{\frac{1}{n-1} \sum_{i=2}^{n}(e(i))^{2},} \\
\text { MAPE }=\frac{1}{n-1} \sum_{i=2}^{n} \frac{|e(i)|}{x^{(0)}(i)} \times 100 \%,
\end{gathered}
$$

where $e(i)$ is the simulative error and $e(i)=\hat{x}^{(0)}(i)-x^{(0)}(i)$. The MAPE criteria of forecasting accuracies of these models are also given in Table 1.

Case 1. The data from paper [29] are taken as an example to verify the novel model, which describes wind turbine capacity of Europe from 2007 to 2017, the data from 2007 to 2015 are used to build prediction models, and the left two samples are used to examine the accuracies of these models. The prediction results by these models are given in Table 2, 
TABLE 1: MAPE criteria for forecasting accuracy.

\begin{tabular}{lcccc}
\hline MAPE (\%) & $<10$ & $10-20$ & $20-50$ & $>50$ \\
\hline Forecasting ability & Excellent & Good & Reasonable & Weak \\
\hline
\end{tabular}

TABLE 2: The results of five grey models in wind turbine capacity of Europe (megawatts).

\begin{tabular}{|c|c|c|c|c|c|c|}
\hline Year & Raw data & $\begin{array}{c}\mathrm{GM}(1,1) \\
a=-0.11 \\
b=58682.19\end{array}$ & $\begin{array}{c}\operatorname{DGM}(1,1) \\
\beta_{1}=1.12 \\
\beta_{2}=62146.52\end{array}$ & $\begin{array}{c}\operatorname{NGBM}(1,1) \\
\gamma=0.10 \\
a=-0.11 \\
b=51933.42\end{array}$ & $\begin{array}{c}\operatorname{NGM}(1,1, k, c) \\
a=-0.04 \\
b=6967.69 \\
c=47718.09\end{array}$ & $\begin{array}{c}\text { ONGM }(1,1, k, c) \\
u=1.19 \\
a=-0.04 \\
b=452.29 \\
c=46501.76\end{array}$ \\
\hline 2007 & 56748.89 & & & & & \\
\hline 2008 & 64943.48 & 68733.07 & 68819.11 & 68053.13 & 62092.57 & 64959.03 \\
\hline 2009 & 77019.99 & 76808.49 & 76910.91 & 76293.36 & 72082.88 & 76284.64 \\
\hline 2010 & 86721.97 & 85832.70 & 85954.16 & 85366.11 & 82532.46 & 86889.65 \\
\hline 2011 & 96603.13 & 95917.15 & 96060.72 & 95432.01 & 93462.41 & 97623.26 \\
\hline 2012 & 109884.87 & 107186.43 & 107355.62 & 106629.26 & 104894.83 & 108856.78 \\
\hline 2013 & 120994.68 & 119779.73 & 119978.58 & 119099.88 & 116852.81 & 120818.76 \\
\hline 2014 & 133915.44 & 133852.61 & 134085.77 & 132997.36 & 129360.50 & 133684.27 \\
\hline 2015 & 147637.65 & 149578.91 & 149851.68 & 148490.51 & 142443.19 & 147607.95 \\
\hline \multicolumn{2}{|c|}{ RMSE } & 1877.41 & 1880.05 & 1909.43 & 4325.85 & 586.52 \\
\hline \multicolumn{2}{|c|}{ MAPE (\%) } & 1.58 & 1.54 & 1.79 & 4.22 & 0.44 \\
\hline 2016 & 161939.87 & 167152.88 & 167471.36 & 165766.36 & 156127.30 & 162739.02 \\
\hline 2017 & 178314.15 & 186791.62 & 187162.78 & 185032.76 & 170440.49 & 179229.35 \\
\hline \multicolumn{2}{|c|}{ RMSE } & 7037.15 & 7378.88 & 5467.26 & 6920.28 & 859.14 \\
\hline \multicolumn{2}{|c|}{ MAPE (\%) } & 3.99 & 4.19 & 3.07 & 4.00 & 0.50 \\
\hline
\end{tabular}

and curves of raw data and simulated and predicted values are graphed in Figure 1.

Case 2. The data from paper [30] are taken as the second example which depicts the carbon dioxide emissions of India from 2000 to 2018. Similar to Case 1, the first 17 data are used to build the prediction models, and the other data are used to examine the prediction accuracies of these models. Table 3 lists the prediction results, and Figure 2 exhibits the curves of raw data and predictive values.

From the above computational results, the desired conclusions can be drawn as follows:

In Table 2, for simulated period, the RMSE values of competing models including $\operatorname{GM}(1,1), \operatorname{DGM}(1,1)$, $\operatorname{NGBM}(1,1), \operatorname{NGM}(1,1, k, c)$, and the proposed model are $1877.41,1880.05,1909.43,4325.85$, and 586.52 ; the MAPE values of these models are $1.58 \%, 1.54 \%, 1.79 \%$, $4.22 \%$, and $0.44 \%$, respectively. For predicted period, the RMSE values of competing models are 7037.15, $7378.88,5467.26,6920.28$, and 859.14; the MAPE values of these models are $3.99 \%, 4.19 \%, 3.07 \%, 4.00 \%$, and $0.50 \%$, respectively. It is seen that the proposed model outperforms among these models owing to its lowest RMSE value and MAPE value either in simulated period or in predicted period. In Figure 1, it is known that simulated and predicted values are more close to actual data.

In Table 3, for simulated period, the RMSE values of these models are $30.83,30.86,31.66,25.37$, and 19.83; the MAPE values of these models are $1.59 \%, 1.60 \%$,

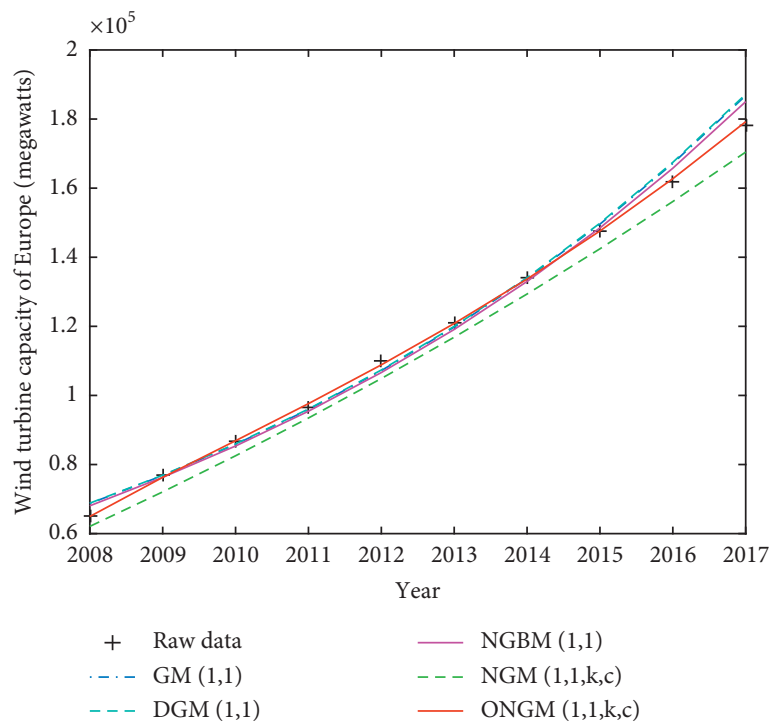

Figure 1: Curves of raw data and predicted values using the five grey system models.

$2.03 \%, 1.50 \%$, and $0.99 \%$, respectively. For predicted period, the RMSE values of these models are 105.93, $107.18,83.74,50.7323$, and 35.25; the MAPE values of them are $4.42 \%, 4.47 \%, 3.48 \%, 1.93 \%$, and $1.09 \%$, respectively. It is obviously found that the proposed model has a better prediction performance with the lowest RMSE values and MAPE values both in simulated period and predicted period. From Figure 2, it is 
TABLE 3: The results of five grey models in carbon dioxide emissions of India (million tons).

\begin{tabular}{|c|c|c|c|c|c|c|}
\hline Year & Raw data & $\begin{array}{l}\mathrm{GM}(1,1) \\
a=-0.06 \\
b=867.30\end{array}$ & $\begin{array}{c}\operatorname{DGM}(1,1) \\
\beta_{1}=1.06 \\
\beta_{2}=894.24\end{array}$ & $\begin{array}{c}\operatorname{NGBM}(1,1) \\
\begin{array}{c}\gamma=0.01 \\
a=-0.06 \\
b=802.75\end{array}\end{array}$ & 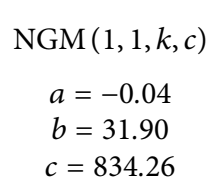 & $\begin{array}{c}\text { ONGM }(1,1, k, c) \\
u=0.73 \\
a=-0.09 \\
b=84.41 \\
c=1011.42\end{array}$ \\
\hline 2000 & 962.5 & & & & & \\
\hline 2001 & 970.3 & 969.25 & 969.62 & 956.09 & 934.76 & 970.10 \\
\hline 2002 & 1021.9 & 1026.60 & 1027.00 & 1016.52 & 1002.39 & 1000.66 \\
\hline 2003 & 1062.3 & 1087.34 & 1087.78 & 1078.93 & 1072.60 & 1060.32 \\
\hline 2004 & 1116.6 & 1151.68 & 1152.15 & 1144.22 & 1145.44 & 1131.70 \\
\hline 2005 & 1204.6 & 1219.83 & 1220.33 & 1212.86 & 1221.03 & 1209.57 \\
\hline 2016 & 1252.5 & 1292.01 & 1292.55 & 1285.19 & 1299.46 & 1291.80 \\
\hline 2007 & 1365.5 & 1368.46 & 1369.04 & 1361.51 & 1380.84 & 1377.38 \\
\hline 2008 & 1466.9 & 1449.43 & 1450.05 & 1442.12 & 1465.28 & 1465.78 \\
\hline 2009 & 1595.6 & 1535.20 & 1535.86 & 1527.29 & 1552.90 & 1556.70 \\
\hline 2010 & 1661.0 & 1626.03 & 1626.79 & 1617.32 & 1643.81 & 1650.01 \\
\hline 2011 & 1735.7 & 1722.24 & 1723.01 & 1712.50 & 1738.15 & 1745.61 \\
\hline 2012 & 1849.2 & 1824.15 & 1824.98 & 1813.15 & 1836.03 & 1843.46 \\
\hline 2013 & 1930.0 & 1932.09 & 1932.98 & 1919.60 & 1937.60 & 1943.56 \\
\hline 2014 & 2083.3 & 2046.41 & 2047.36 & 2032.19 & 2042.96 & 2045.91 \\
\hline 2015 & 2147.8 & 2167.50 & 2168.52 & 2151.29 & 2152.34 & 2150.54 \\
\hline 2016 & 2234.2 & 2295.75 & 2296.85 & 2277.28 & 2265.80 & 2257.47 \\
\hline \multicolumn{2}{|c|}{ RMSE } & 30.83 & 30.86 & 31.66 & 25.37 & 19.83 \\
\hline \multicolumn{2}{|c|}{ MAPE $(\%)$} & 1.59 & 1.60 & 2.03 & 1.50 & 0.99 \\
\hline 2017 & 2316.9 & 2431.59 & 2432.77 & 2410.57 & 2383.53 & 2366.75 \\
\hline 2018 & 2479.1 & 2575.47 & 2576.73 & 2551.58 & 2505.70 & 2478.42 \\
\hline \multicolumn{2}{|c|}{ RMSE } & 105.93 & 107.18 & 83.74 & 50.7323 & 35.25 \\
\hline \multicolumn{2}{|c|}{ MAPE (\%) } & 4.42 & 4.47 & 3.48 & 1.93 & 1.09 \\
\hline
\end{tabular}

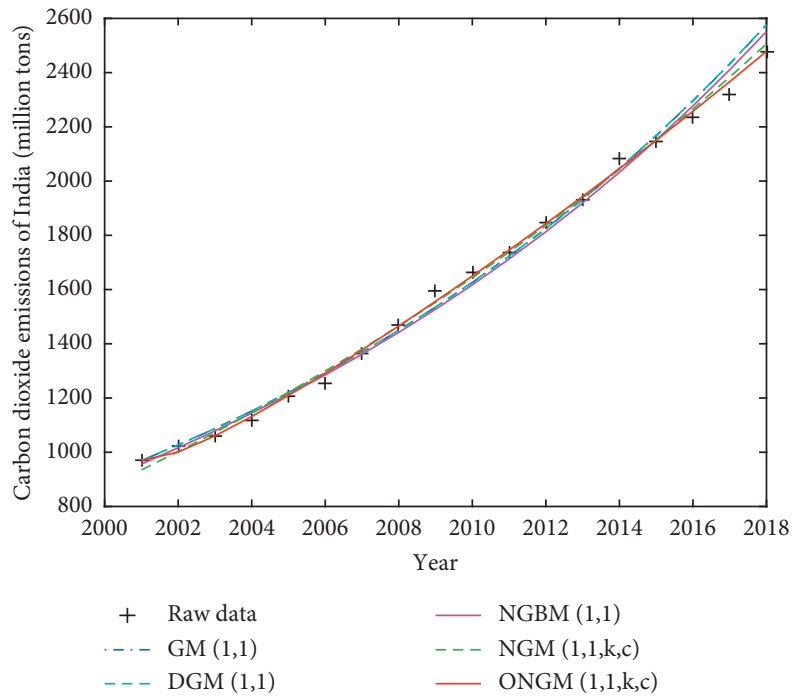

Figure 2: Curves of raw data and predicted values using the five grey system models.

easily seen that the predicted values of the carbon dioxide emissions of India are more close to actual data.

In summary, the proposed model outperforms among these competing models listed in this paper comparing the prediction accuracies of these models. Due to the feasibility of the proposed model, it will be used to predict China's natural gas demand in the next section.

\section{Application}

Natural gas plays an important role in the energy market on account of its high-quality, efficacy, and low-carbon feature. Therefore, development of natural gas has been written in the 13th Five Plan of China which is the most important official policy in China.

This paper collects the data of natural gas demand of China from 2001 to 2013 (cf. [28]), as shown in Table 4. The first ten data are used for building the novel model and other competing models, and the left 2 samples are used to examine the prediction accuracies of these models.

Furthermore, the simulated and predicted values of natural gas demand of China and statistical indices by the five grey models are listed in Table 5 .

Ignoring the first item of predicted value, the simulated and predicted values are shown in Table 5, and these predicted values are graphed in Figure 3. It should be notable that the RMSE value of competitive models including $\operatorname{GM}(1,1), \operatorname{DGM}(1,1), \operatorname{NGBM}(1,1), \operatorname{NGM}(1,1, k, c)$, and the novel model is $30.83,30.86,31.66,25.37$, and 19.83; the MAPE values of these models are $1.59 \%, 1.60 \%, 2.03 \%$, $1.50 \%$, and $0.99 \%$, respectively. What is more, the forecasting ability in predicted period is fact more important; for the predicted stage, the RMSE values of these models are $15.07,15.30,13.91,3.40$, and 2.80; the MAPE values of them are $13.19 \%, 13.39 \%, 12.18 \%, 2.24 \%$, and $1.97 \%$, respectively. It is obviously seen that the proposed model has best prediction performance than that of other grey system models. From the perspective of plotting curves of raw data and 
TABle 4: Natural gas demand of China from 2003 to 2013 (billion cubic meters).

\begin{tabular}{lccc}
\hline Year & Raw data & Year & Raw data \\
\hline 2003 & 35.0 & 2009 & 85.2 \\
2004 & 41.5 & 2010 & 94.8 \\
2005 & 49.3 & 2011 & 103.1 \\
2016 & 58.6 & 2012 & 107.2 \\
2007 & 69.2 & 2013 & 119.3 \\
2008 & 80.3 & & \\
\hline
\end{tabular}

TABLE 5: The results of five grey models in natural gas demand of China (billion cubic meters).

\begin{tabular}{|c|c|c|c|c|c|c|}
\hline Year & Raw data & $\begin{array}{c}\mathrm{GM}(1,1) \\
a=-0.11 \\
b=9723.73\end{array}$ & $\begin{array}{c}\operatorname{DGM}(1,1) \\
\beta_{1}=1.12 \\
\beta_{2}=10312.95\end{array}$ & $\begin{array}{c}\operatorname{NGBM}(1,1) \\
\gamma=0.05 \\
a=-0.10 \\
b=5987.40\end{array}$ & $\begin{array}{l}\operatorname{NGM}(1,1, k, c) \\
\begin{array}{c}a=0.08 \\
b=3477.01 \\
c=3169.31\end{array}\end{array}$ & $\begin{array}{c}\text { ONGM }(1,1, k, c) \\
u=1.03 \\
a=0.07 \\
b=3155.37 \\
c=4964.02\end{array}$ \\
\hline 2003 & 35.0 & & & & & \\
\hline 2004 & 41.5 & 46.18 & 46.29 & 45.74 & 35.15 & 40.69 \\
\hline 2005 & 49.3 & $52.07 \mathrm{GM}(1,1)$ & 52.19 & 51.73 & 45.04 & 50.32 \\
\hline 2016 & 58.6 & 58.70 & 58.83 & 58.40 & 54.65 & 59.78 \\
\hline 2007 & 69.2 & 66.19 & 66.32 & 65.87 & 63.98 & 68.95 \\
\hline 2008 & 80.3 & 74.61 & 74.77 & 74.24 & 73.03 & 77.85 \\
\hline 2009 & 85.2 & 84.12 & 84.30 & 83.66 & 81.83 & 86.50 \\
\hline 2010 & 94.8 & 94.84 & 95.02 & 94.24 & 90.36 & 94.92 \\
\hline 2011 & 103.1 & 106.92 & 107.13 & 106.16 & 98.65 & 103.13 \\
\hline \multicolumn{2}{|c|}{ RMSE } & 3.29 & 3.30 & 3.23 & 5.06 & 1.17 \\
\hline \multicolumn{2}{|c|}{ MAPE (\%) } & 4.19 & 4.26 & 4.15 & 7.53 & 1.39 \\
\hline 2012 & 107.2 & 120.55 & 120.77 & 119.55 & 106.70 & 111.14 \\
\hline 2013 & 119.3 & 135.91 & 136.15 & 134.62 & 114.52 & 118.98 \\
\hline \multicolumn{2}{|c|}{ RMSE } & 15.07 & 15.30 & 13.91 & 3.40 & 2.80 \\
\hline \multicolumn{2}{|c|}{ MAPE (\%) } & 13.19 & 13.39 & 12.18 & 2.24 & 1.97 \\
\hline
\end{tabular}

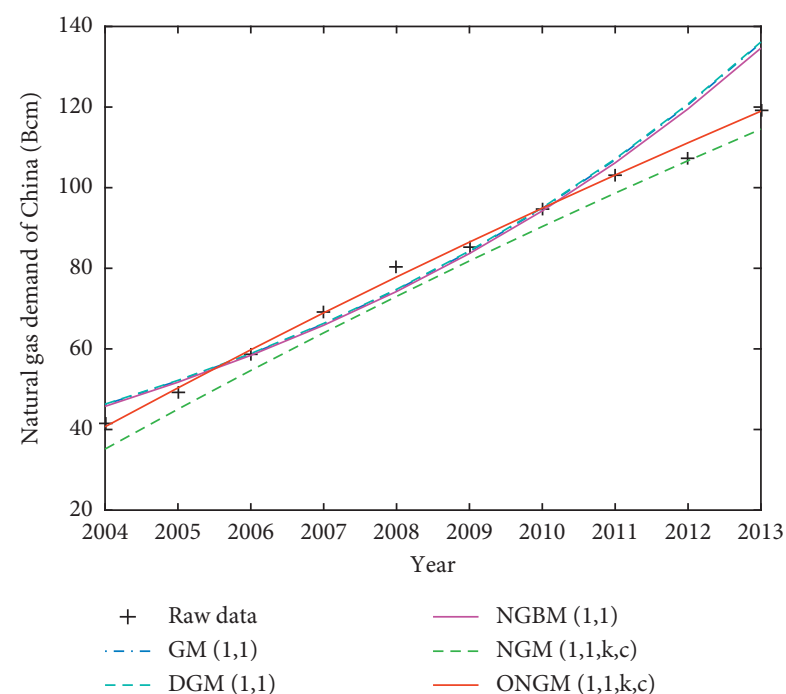

Figure 3: Curves of raw data and predicted values using the five grey system models.

simulated and predicted values, it is shown that the fitting values of the proposed model are more close to actual data. Therefore, the proposed model can improve the prediction performance significantly and should be regarded as an appreciate method to predict natural gas demand of China.

\section{Conclusion}

Aimed to improve further the prediction performance of the nonhomogeneous grey model, a new whitening equation with variable coefficient is introduced into the nonhomogeneous grey model; as a result of this paper, $\operatorname{ONGM}(1,1, k, c)$ is proposed. The main contributions of this paper can be summarized as follows:

(1) A new whitening equation with variable coefficient is introduced into the traditional nonhomogeneous grey model to further generalize the mathematical form of the nonhomogeneous grey model.

(2) By using grey modelling techniques, the time response function of the novel model and restored values of the original series are deduced in detail.

(3) Two empirical examples are used to prove the validity of the novel, and the novel model is applied to predict natural gas demand of China. The results indicate that the novel model has a better prediction performance compared with other competitive models.

Up to this point, the advantages of the proposed model have been discussed. However, there still exist some issues, which we should concentrate and solve. For instance, the $\operatorname{ONGM}(1,1, k, c)$ model is regarded as a kind of univariate 
model, that is, the other influential factors could be ignored in applications, and the multivariable model with shape parameter will be discussed in our future work; the fractional-order accumulation should also be introduced in this study to further enhance the prediction performance.

\section{Data Availability}

Raw data used for this work can be found in the existing literature.

\section{Conflicts of Interest}

The authors declare that there are no conflicts of interest regarding the publication of this paper.

\section{Acknowledgments}

This work was supported by the National Natural Science Foundation of China (Grant no. 11661001) and the Natural Science Foundation of Guangxi (Grant no. 2016GXNSFBA380069).

\section{References}

[1] J.-L. Deng, "Control problems of grey systems," Systems \& Control Letters, vol. 1, no. 5, pp. 288-294, 1982.

[2] Q. Wang, L. Liu, S. Wang, J.-Z. Wang, and M. Liu, "Predicting Beijing's tertiary industry with an improved grey model," Applied Soft Computing, vol. 57, pp. 482-494, 2017.

[3] G. M. Duman, E. Kongar, and S. M. Gupta, "Estimation of electronic waste using optimized multivariate grey models," Waste Management, vol. 95, pp. 241-249, 2019.

[4] J. Kang and H. Zhao, "Application of improved grey model in long-term load forecasting of power engineering," Systems Engineering Procedia, vol. 3, pp. 85-91, 2012.

[5] X. Guo, S. Liu, L. Wu, Y. Gao, and Y. Yang, "A multi-variable grey model with a self-memory component and its application on engineering prediction," Engineering Applications of Artificial Intelligence, vol. 42, pp. 82-93, 2015.

[6] S. Bahrami, R.-A. Hooshmand, and M. Parastegari, "Short term electric load forecasting by wavelet transform and grey model improved by PSO (particle swarm optimization) algorithm," Energy, vol. 72, pp. 434-442, 2014.

[7] H. Mostafaei and S. Kordnoori, "Hybrid grey forecasting model for Irans energy consumption and supply," International Journal of Energy Economics and Policy, vol. 2, no. 3, pp. 97-102, 2012.

[8] F. Shaikh, Q. Ji, P. H. Shaikh, N. H. Mirjat, and M. A. Uqaili, "Forecasting China's natural gas demand based on optimised nonlinear grey models," Energy, vol. 140, pp. 941-951, 2017.

[9] N.-m. Xie and S.-f. Liu, "Discrete grey forecasting model and its optimization," Applied Mathematical Modelling, vol. 33, no. 2, pp. 1173-1186, 2009.

[10] U. Kumar and V. K. Jain, “Time series models (Grey-Markov, Grey Model with rolling mechanism and singular spectrum analysis) to forecast energy consumption in India," Energy, vol. 35, no. 4, pp. 1709-1716, 2010.

[11] C. Hamzacebi and H. A. Es, "Forecasting the annual electricity consumption of Turkey using an optimized grey model," Energy, vol. 70, pp. 165-171, 2014.
[12] S. Ding, K. W. Hipel, and Y.-G. Dang, "Forecasting China's electricity consumption using a new grey prediction model," Energy, vol. 149, pp. 314-328, 2018.

[13] Y.-Y. Chen, H.-T. Liu, and H.-L. Hsieh, "Time series interval forecast using GM $(1,1)$ and NGBM $(1,1)$ models," Soft Computing, vol. 23, no. 5, pp. 1541-1555, 2019.

[14] W.-Z. Wu and T. Zhang, "An improved gray interval forecast method and its application," Communications in Statistics Theory and Methods, vol. 49, no. 5, pp. 1120-1131, 2020.

[15] X. Ma and Z. Liu, "Application of a novel time-delayed polynomial grey model to predict the natural gas consumption in China," Journal of Computational and Applied Mathematics, vol. 324, pp. 17-24, 2017.

[16] R. Intharathirat, P. Abdul Salam, S. Kumar, and A. Untong, "Forecasting of municipal solid waste quantity in a developing country using multivariate grey models," Waste Management, vol. 39, pp. 3-14, 2015.

[17] C.-I. Chen, H. L. Chen, and S.-P. Chen, "Forecasting of foreign exchange rates of Taiwan's major trading partners by novel nonlinear Grey Bernoulli model NGBM $(1,1)$," Communications in Nonlinear Science and Numerical Simulation, vol. 13, no. 6, pp. 1194-1204, 2008.

[18] Z.-X. Wang, K. W. Hipel, Q. Wang, and S.-W. He, “An optimized NGBM $(1,1)$ model for forecasting the qualified discharge rate of industrial wastewater in China," Applied Mathematical Modelling, vol. 35, no. 12, pp. 5524-5532, 2011.

[19] W.-Z. Wu, T. Zhang, and C.-L. Zheng, "A novel optimized nonlinear grey Bernoulli model for forecasting China’s GDP," Complexity, vol. 2019, Article ID 1731262, 10 pages, 2019.

[20] N. T. Nguyen, V. T. Phan, and Z. Malarad, "Nonlinear grey Bernoulli model based on fourier transformation and its application in forecasting the electricity consumption in Vietnam," Journal of Intelligent \& Fuzzy Systems, vol. 37, no. 6, pp. 7631-7641, 2019.

[21] X. Ma, Z. Liu, and Y. Wang, "Application of a novel nonlinear multivariate grey Bernoulli model to predict the tourist income of China," Journal of Computational and Applied Mathematics, vol. 347, pp. 84-94, 2019.

[22] L. Wu, S. Liu, L. Yao, S. Yan, and D. Liu, "Grey system model with the fractional order accumulation," Communications in Nonlinear Science and Numerical Simulation, vol. 18, no. 7, pp. 1775-1785, 2013.

[23] L. Wu, X. Gao, Y. Xiao, Y. Yang, and X. Chen, "Using a novel multi-variable grey model to forecast the electricity consumption of Shandong Province in China," Energy, vol. 157, pp. 327-335, 2018.

[24] X. Ma, W. Wu, B. Zeng, Y. Wang, and X. Wu, "The conformable fractional grey system model," ISA Transactions, vol. 96, pp. 255-271, 2020.

[25] J. Cui, S.-F. Liu, B. Zeng, and N.-M. Xie, "A novel grey forecasting model and its optimization," Applied Mathematical Modelling, vol. 37, no. 6, pp. 4399-4406, 2013.

[26] P.-Y. Chen and H. M. Yu, "Foundation settlement prediction based on a novel NGM model," Mathematical Problems in Engineering, vol. 2014, Article ID 242809, 8 pages, 2014.

[27] X. Ma, Y.-S. Hu, and Z.-B. Liu, "A novel kernel regularized nonhomogeneous grey model and its applications," Communications in Nonlinear Science and Numerical Simulation, vol. 48, pp. 51-62, 2017.

[28] B. Zeng and S. Liu, "A self-adaptive intelligence gray prediction model with the optimal fractional order accumulating operator and its application," Mathematical Methods in the Applied Sciences, vol. 40, no. 18, pp. 7843-7857, 2017. 
[29] J. Xia, X. Ma, W. Wu, B. Huang, and W. Li, "Application of a new information priority accumulated grey model with time power to predict short-term wind turbine capacity," Journal of Cleaner Production, vol. 244, Article ID 118573, 2020.

[30] W. Wu, X. Ma, Y. Zhang, W. Li, and Y. Wang, "A novel conformable fractional non-homogeneous grey model for forecasting carbon dioxide emissions of BRICS countries," Science of the Total Environment, vol. 707, Article ID 135447, 2020. 